\title{
Combining the Remote Microphone Technique with Head-tracking for Local Active Sound Control
}

\author{
Woomin Jung,* Stephen J. Elliott, and Jordan Cheer \\ Institute of Sound and Vibration Research, \\ University of Southampton, SO17 1BJ, United Kingdom
}

(Dated: June 9, 2017)

\begin{abstract}
This paper describes practical integration of the remote microphone technique with a headtracking device in a local active noise control system. The formulation is first reviewed for the optimized observation filter and nearfield pressure estimation. The attenuation performance and stability of an adaptive active headrest system combined with the remote microphone technique are then studied. The accuracy of the nearfield estimation and the effect of the head-tracking on the control performance are investigated in real-time experiments. The regularization factor of the observation filter is selected as a trade-off between its accuracy and its robustness. The integrated active headrest system is used to estimate and attenuate disturbance signals at a listener's ears from a single tonal primary source, while a commercial head-tracking device detects and provides the real-time head position to the active headrest system whose responses are updated accordingly.
\end{abstract}

PACS numbers: PACS: 43.38.-p, 43.50.Ki

\footnotetext{
*wj3e13@soton.ac.uk; Corresponding author.
} 


\section{INTRODUCTION}

Active sound control in enclosures can be mainly divided into two strategies: global active control and local active control [1] [2] [3]. Global active control is designed to reduce the overall acoustic potential energy in an enclosure, and previous research has demonstrated that the attenuation performance of global active control is generally limited to low frequencies. For instance, global control inside a car cabin sized enclosure can be effective at frequencies below about $300 \mathrm{~Hz}$, but because the modal density increases at higher frequencies, it is difficult to achieve control using a limited number of loudspeakers [2]. In contrast, since local active control aims to reduce the sound pressure at particular locations, a higher level of attenuation can be achieved at higher frequencies. Zones of quiet are thus generated at the control-targeted locations such as a listener's ears in a vehicle cabin. Previous research has investigated the use of active headrest systems to achieve local active control in a practical vehicle cabin [4] [5] [6] [7]. In the active headrest system, secondary control sources and error sensors are installed around the headrests of the seats to control the interior noise around the driver and passengers.

Although the attenuation performance of the active headrest system depends on various factors, including the geometry of the active headrest system and the acoustic characteristics of the sound field, it is demonstrated that for a single channel system in a diffuse field, the $10 \mathrm{~dB}$ zone of quiet, in which at least $10 \mathrm{~dB}$ of attenuation is achieved, is formed around the error microphone with a diameter of about one tenth of the acoustic wavelength [8]. At higher frequencies the $10 \mathrm{~dB}$ zone of quiet becomes increasingly small. Additionally, when the physical error microphones are remotely installed from control-targeted areas due to practical limitations, the $10 \mathrm{~dB}$ zone of quiet may be generated outside of the controltargeted areas. To overcome this limitation, a number of virtual sensing algorithms have been proposed [9] [10] [11]. The virtual sensing algorithms allow the signals at virtual error microphones, which are located in the control-targeted areas, to be estimated from a set of physical monitoring microphones, which are installed remotely from the target areas. Amongst the virtual sensing algorithms, the remote microphone technique, initially suggested by Roure and Albarrazin [9], assumes that a filter, called the observation filter here, can be used to estimate the disturbance signals at the virtual error sensors from the disturbance signals at the monitoring sensors. A formulation for the optimal design of 
this observation filter in the frequency domain is presented by Elliott and Cheer [11]. The present paper investigates the accuracy and robustness of the remote microphone technique in an active headrest, to estimate the disturbance signals at the listener's ears from the disturbance signals at the monitoring sensors on the headrest.

Another practical issue concerning local active control is the effect of listener head movements on the control performance. When local control is implemented around a listener's ears, head movements during control can introduce changes to the sound field and the acoustic responses. In particular, if the remote microphone technique is applied, the accuracy of the virtual sensing estimation method can be degraded by head movement and as a result, the attenuation performance and stability of the adaptive algorithm can be reduced. To overcome this problem, moving virtual sensing methods have been studied [12] [13] and the application of a head-tracking device to local active control has also been suggested [10] [14] [15]. That is, as the head-tracking device detects a change in the head position, this information can be utilized to update the controller and the observation filter using pre-calculated responses, which are required to implement an adaptive local active control system. This paper goes beyond previous conference publications [14] [15] in its discussions of how the remote microphone technique can be used in practice and on the condition for the stability of the overall tonal adaptive control system with integrated head-tracking. Moreover, a complete experimental implementation, which includes practical head-tracking, is presented and validates the performance and stability analysis.

In Section II, a theoretical analysis of the remote microphone technique and the integrated active headrest system are presented. In Section III, the real-time implementation of the nearfield estimation and integrated active headrest system for controlling tonal sounds is presented. Finally, in Section IV, conclusions are drawn.

\section{THE COMBINED LOCAL ACTIVE CONTROL SYSTEM}

In this section, the remote microphone technique is reviewed using a frequency-domain formulation to obtain the optimized observation filter. In addition, for the case when the remote microphone technique is combined with an adaptive feedforward active control system, the optimized control signals and attenuation performance are also formulated. Finally, a condition for the stability of the combined system is derived. Analysis in the frequency 
domain allows a straightforward assessment of both the nearfield estimation and the behaviour of the active control system when it aims to control tonal noise. For generality, all signals are initially assumed to be stationary random, so that only their spectral densities are time-invariant [11], but their frequency dependence is suppressed for notational convenience.

\section{A. The remote microphone technique for nearfield estimation}

As discussed in the introduction, it is often impractical to install physical error microphones close to the ears of the listener. To overcome this problem, the remote microphone technique [9] [10] [11] can be applied to estimate virtual error signals from the signals measured at the remote monitoring microphones, as shown in Fig. 1. The remote microphone technique relies on the primary field being sufficiently uniform that there is a strong spatial correlation between the monitoring microphones and the virtual error microphones.

The primary sources produce a vector of complex disturbance signals, at a given frequency, of $\mathbf{d}_{e}=\left[\begin{array}{llll}d_{e 1}, & d_{e 2} & \ldots & d_{e N e}\end{array}\right]^{T}$ at the virtual microphones, and the secondary sources are driven by the vector of control signals $\mathbf{u}=\left[\begin{array}{llll}u_{1}, u_{2} & \ldots & u_{N u}\end{array}\right]^{T}$ to minimize these disturbance signals. The vector of complex error signals, e, at the virtual microphones after control can be defined as

$$
\mathbf{e}=\mathbf{d}_{e}+\mathbf{G}_{e} \mathbf{u},
$$

where $\mathbf{G}_{e}$ is the matrix of plant responses between the secondary sources and the virtual error microphones. When the direct measurement of the physical error signals is infeasible, they can be estimated from the monitoring microphone signals $\mathbf{m}=\left[\begin{array}{llll}m_{1}, m_{2} & \ldots & m_{N m}\end{array}\right]^{T}$, which are defined as

$$
\mathbf{m}=\mathbf{d}_{m}+\mathbf{G}_{m} \mathbf{u},
$$

where $\mathbf{d}_{m}$ is the vector of disturbance signals at the monitoring microphones, which is described as $\mathbf{d}_{m}=\left[\begin{array}{llll}d_{m 1}, & d_{m 2} & \ldots & d_{m N m}\end{array}\right]^{T}$ and $\mathbf{G}_{m}$ is the matrix of plant responses between the secondary sources and the monitoring microphones. The remote microphone technique is used, as illustrated in Fig. 1, where it is assumed that only estimates of the 


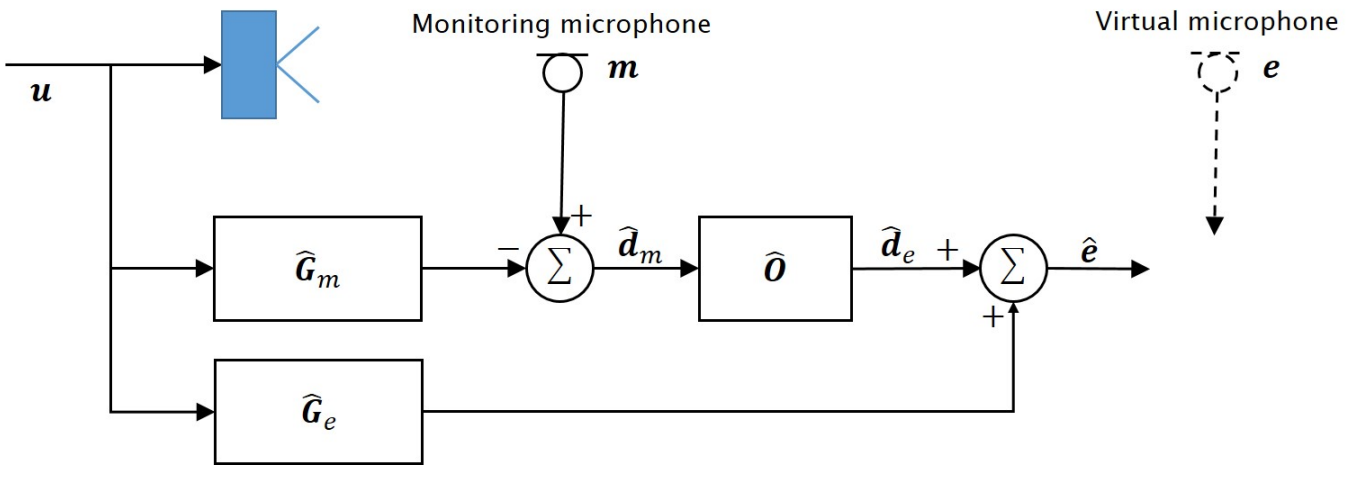

FIG. 1. Block diagram of the remote microphone technique.

plant responses $\mathbf{G}_{e}$ and $\mathbf{G}_{m}$ are available, as denoted by $\hat{\mathbf{G}}_{e}$ and $\hat{\mathbf{G}}_{m}$, and $\hat{\mathbf{O}}$ is the estimated observation filter used to calculate $\hat{\mathbf{d}}_{e}$ from $\hat{\mathbf{d}}_{m}$. The estimated error signals, $\hat{\mathbf{e}}$, at the virtual error microphones can then be written as

$$
\hat{\mathbf{e}}=\hat{\mathbf{d}}_{e}+\hat{\mathbf{G}}_{e} \mathbf{u}=\hat{\mathbf{O}} \hat{\mathbf{d}}_{m}+\hat{\mathbf{G}}_{e} \mathbf{u}=\hat{\mathbf{O}}\left(\mathbf{m}-\hat{\mathbf{G}}_{m} \mathbf{u}\right)+\hat{\mathbf{G}}_{e} \mathbf{u} .
$$

The observation filter in Eq. (3) will influence how accurately $\hat{\mathbf{d}}_{e}$ is estimated, compared to $\mathbf{d}_{e}$ and the estimation error between them is an important factor for the performance of the control system. The optimal observation filter, $\mathbf{O}_{\text {opt }}$, can be derived by minimizing the estimation error between $\mathbf{d}_{e}$ and $\hat{\mathbf{d}}_{e}$. In practice, however, it is also necessary to include a term in the cost function that is proportional to the effort required by the observation filter to improve the robustness of this filter. If $\mathbf{d}_{e}$ and $\mathbf{d}_{m}$ are measured in preliminary experiments before active control and $\mathbf{d}_{m}$ is thus identical to $\hat{\mathbf{d}}_{m}, \mathbf{O}_{\text {opt }}$ can be obtained by minimizing the cost function [11],

$$
J_{1}=\operatorname{trace}\left\{E\left[\left(\mathbf{d}_{e}-\mathbf{O d}_{m}\right)\left(\mathbf{d}_{e}-\mathbf{O d}_{m}\right)^{H}+\beta \mathbf{O O}^{H}\right]\right\},
$$

where $E[]$ is the expectation operator and ${ }^{H}$ is the Hermitian, complex conjugate transpose and $\beta$ is a positive real effort-weighting parameter. In the calculation of the optimized filter it is assumed that $\mathbf{d}_{e}$ and $\mathbf{d}_{m}$ are known perfectly. From Eq. (4), when the number of monitoring microphones $(\mathrm{Nm})$ is larger than the number of error microphones $(\mathrm{Ne})$, the problem is mathematically overdetermined and $\mathbf{O}_{\text {opt }}$, which minimizes $J_{1}$ in Eq. (4), can be calculated using similar methods to those described in Ref. [2] to give 


$$
\mathbf{O}_{\mathrm{opt}}=\mathbf{S}_{d_{m} d_{e}}\left(\mathbf{S}_{d_{m} d_{m}}+\beta \mathbf{I}\right)^{-1}=\mathbf{P}_{e} \mathbf{S}_{v v} \mathbf{P}_{m}^{H}\left(\mathbf{P}_{m} \mathbf{S}_{v v} \mathbf{P}_{m}^{H}+\beta \mathbf{I}\right)^{-1},
$$

where $\mathbf{S}_{d_{m} d_{e}}=E\left[\mathbf{d}_{e} \mathbf{d}_{m}^{H}\right]$ is the cross spectral density matrix between $\mathbf{d}_{m}$ and $\mathbf{d}_{e}, \mathbf{S}_{d_{m} d_{m}}=$ $E\left[\mathbf{d}_{m} \mathbf{d}_{m}^{H}\right]$ is the power spectral density matrix for $\mathbf{d}_{m}, \mathbf{I}$ is the identity matrix having the same dimensions as $\mathbf{S}_{d_{m} d_{m}}$ and the leakage term, $\beta$, is seen to act as a regularization factor. If $\mathbf{S}_{v v}$ is the power spectral density matrix of primary source strengths, $\mathbf{v}$, and $\mathbf{P}_{e}$ and $\mathbf{P}_{m}$ are the matrices of acoustical transfer responses from the primary sources to the error microphones and monitoring microphones respectively, $\mathbf{O}_{\text {opt }}$ can be written in the alternative form on the right-hand side of Eq. (5). When practical estimates of $\mathbf{P}_{e}$ and $\mathbf{P}_{m}$ are used to calculate the observation filter, it is denoted $\hat{\mathbf{O}}_{\text {opt }}$ and applied to Eq. (3).

When the inverse term in Eq. (5) has a large condition number, $\mathbf{O}_{\text {opt }}$ can be sensitive to physical and numerical uncertainties. A reduction in the condition number can be obtained by appropriate selection of the regularization factor, $\beta$. Although the solution for the optimal observation filter with regularization becomes more robust to practical uncertainties, an excessively large regularization factor can produce a biased solution with a higher estimation error. Therefore, to select an appropriate regularization factor, it is necessary to consider both the robustness and the accuracy of the solution.

\section{B. Local active control combined with the remote microphone technique and} head-tracking

To use the remote microphone technique in practice it is combined with the filteredreference LMS algorithm for feedforward control, and the block diagram of the combined algorithm is shown in Fig. 2. In Fig. 2, the error signals estimated using the remote microphone technique with the optimal observation filter are multiplied by the filtered-reference signals to update the control filter coefficients. The active control system thus attempts to

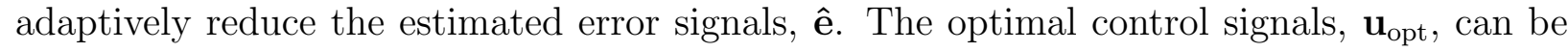
obtained by minimizing ê using the cost function defined as

$$
J_{2}=\operatorname{trace}\left\{E\left[\hat{\mathbf{e}} \hat{\mathbf{e}}^{H}\right]\right\} .
$$

To describe Eq. (6) in terms of $\mathbf{u}$, we first substitute Eq. (2) into Eq. (3) and use the optimal 
estimated observation filter, $\hat{\mathrm{O}}_{\text {opt }}$, so Eq. (3) can be expressed as

$$
\hat{\mathbf{e}}=\hat{\mathbf{O}}_{\mathrm{opt}} \mathbf{d}_{m}+\left[\hat{\mathbf{G}}_{e}+\hat{\mathbf{O}}_{\mathrm{opt}}\left(\mathbf{G}_{m}-\hat{\mathbf{G}}_{m}\right)\right] \mathbf{u} \text {. }
$$

In Eq. (7), the term, $\hat{\mathbf{G}}_{e}+\hat{\mathbf{O}}_{\text {opt }}\left(\mathbf{G}_{m}-\hat{\mathbf{G}}_{m}\right)$ can be defined as the effective plant response, $\mathbf{G}$, between $\mathbf{u}$ and $\hat{\mathbf{e}}$, and only if both $\hat{\mathbf{G}}_{e}$ is equal to $\mathbf{G}_{e}$ and $\hat{\mathbf{G}}_{m}$ is equal to $\mathbf{G}_{m}$, will this plant response be equal $\mathbf{G}_{e}$. By substituting Eq. (7) into Eq. (6), the cost function can be written as

$$
J_{2}=\operatorname{trace}\left\{E\left[\left(\hat{\mathbf{O}}_{\mathrm{opt}} \mathbf{d}_{m}+\mathbf{G u}\right)\left(\hat{\mathbf{O}}_{\mathrm{opt}} \mathbf{d}_{m}+\mathbf{G u}\right)^{H}\right]\right\} .
$$

The optimal control signals, $\mathbf{u}_{\mathrm{opt}}$, that minimize Eq. (8) can be obtained using the derivation detailed in Ref. [2] as

$$
\mathbf{u}_{\mathrm{opt}}=-\left(\mathbf{G}^{H} \mathbf{G}\right)^{-1} \mathbf{G}^{H} \hat{\mathbf{O}}_{\mathrm{opt}} \mathbf{d}_{m}
$$

The spectral density matrix of the signals at the error microphones, $\mathbf{S}_{e e}$, after optimal control can then be obtained by substituting Eq. (9) into Eq. (1), so that

$$
\begin{aligned}
\mathbf{S}_{e e}= & E\left[\left(\mathbf{d}_{e}+\mathbf{G}_{e} \mathbf{u}_{\mathrm{opt}}\right)\left(\mathbf{d}_{e}+\mathbf{G}_{e} \mathbf{u}_{\mathrm{opt}}\right)^{H}\right] \\
= & \mathbf{S}_{d_{e} d_{e}}-\mathbf{G}_{e}\left(\mathbf{G}^{H} \mathbf{G}\right)^{-1} \mathbf{G}^{H} \hat{\mathbf{O}}_{\mathrm{opt}} \mathbf{S}_{d_{m} d_{e}}-\mathbf{S}_{d_{m} d_{e}} \hat{\mathbf{O}}_{\mathrm{opt}}^{H} \mathbf{G}\left(\mathbf{G} \mathbf{G}^{H}\right)^{-1} \mathbf{G}_{e}^{H} \\
& +\mathbf{G}_{e}\left(\mathbf{G}^{H} \mathbf{G}\right)^{-1} \mathbf{G}^{H} \hat{\mathbf{O}}_{\mathrm{opt}} \mathbf{S}_{d_{m} d_{m}} \hat{\mathbf{O}}_{\mathrm{opt}}^{H} \mathbf{G}\left(\mathbf{G} \mathbf{G}^{H}\right)^{-1} \mathbf{G}_{e}^{H} .
\end{aligned}
$$

The optimal attenuation performance at the error microphones can then be obtained by dividing the sum of diagonal terms of $\mathbf{S}_{e e}$ by the corresponding term for $\mathbf{S}_{d_{e} d_{e}}$.

To investigate the conditions for the convergence of the adaptive algorithm in Fig. 2, when a single tonal reference signal is assumed, the vector of complex control signals, $\mathbf{u}$, at the $(n+1)$-th iteration can be written as

$$
\mathbf{u}(n+1)=\mathbf{u}(n)-\alpha \hat{\mathbf{G}}_{e}^{H} \hat{\mathbf{e}}(n),
$$

where $\alpha$ is the convergence coefficient and $\hat{\mathbf{e}}(n)$ is the vector of sampled estimated error signals at the $n$th sample time. In Eq. (11), the internal model, $\hat{\mathbf{G}}_{e}$, is applied instead of 


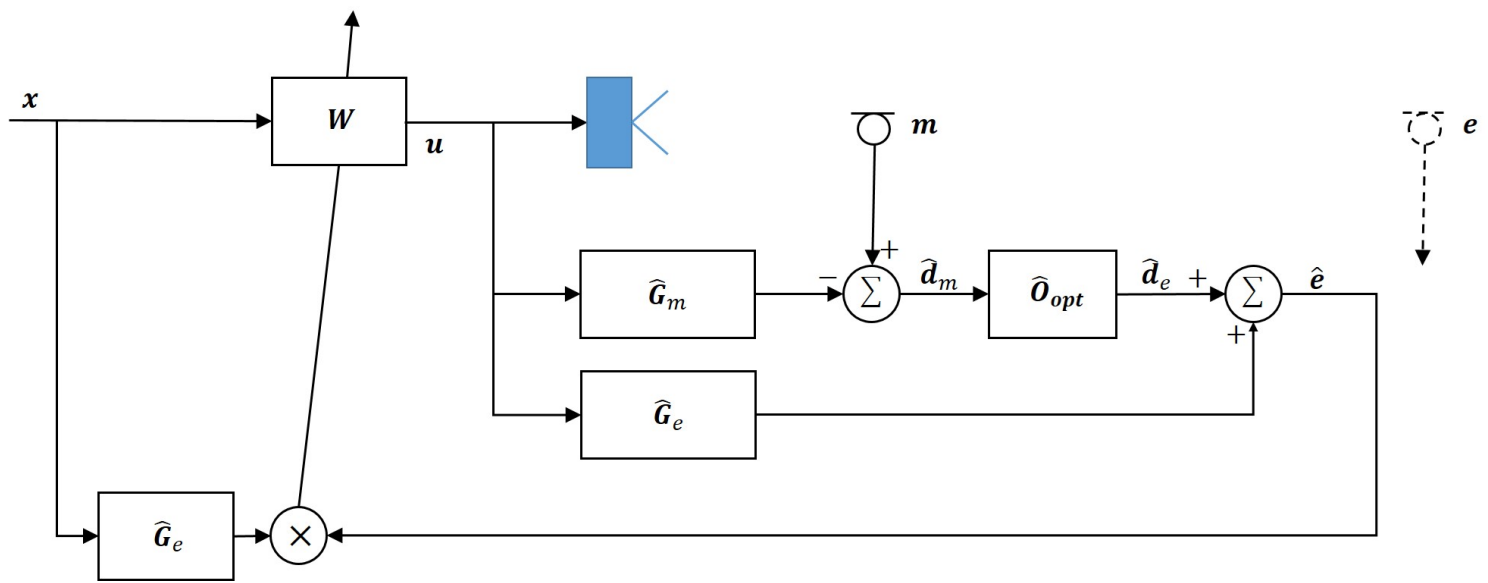

FIG. 2. Block diagram of the filtered-reference LMS algorithm for adaptive feedforward control, combined with the remote microphone technique.

the effective plant response, $\mathbf{G}$, since the difference between $\mathbf{G}_{m}$ and $\hat{\mathbf{G}}_{m}$ is not known and so is assumed to be zero. From Eq. (7), the vector of sampled estimated error signals at the $n$th sample time, $\hat{\boldsymbol{e}}(n)$, can be written as

$$
\hat{\mathbf{e}}(n)=\hat{\mathbf{O}}_{\mathrm{opt}} \mathbf{d}_{m}(n)+\left[\hat{\mathbf{G}}_{e}+\hat{\mathbf{O}}_{\mathrm{opt}}\left(\mathbf{G}_{m}-\hat{\mathbf{G}}_{m}\right)\right] \mathbf{u}(n)=\hat{\mathbf{O}}_{\mathrm{opt}} \mathbf{d}_{m}(n)+\mathbf{G u}(n) .
$$

By substituing Eq. (12) into Eq. (11), Eq. (11) can be expressed as

$$
\mathbf{u}(n+1)=\mathbf{u}(n)-\alpha\left[\hat{\mathbf{G}}_{e}^{H} \hat{\mathbf{O}}_{\mathrm{opt}} \mathbf{d}_{m}(n)+\hat{\mathbf{G}}_{e}^{H} \mathbf{G u}(n)\right]
$$

The expression in square brackets in Eq. (13) is zero after convergence if convergence is achieved. Therefore, the steady state vector of control signals after the convergence, $\mathbf{u}_{\infty}$, is defined to be

$$
\mathbf{u}_{\infty}=-\left(\hat{\mathbf{G}}_{e}^{H} \mathbf{G}\right)^{-1} \hat{\mathbf{G}}_{e}^{H} \hat{\mathbf{O}}_{\mathrm{opt}} \mathbf{d}_{m}
$$

By substracting Eq. (14) from both sides of Eq. (13), Eq. (13) can be written as

$$
\left(\mathbf{u}(n+1)-\mathbf{u}_{\infty}\right)=\left[\mathbf{I}-\alpha \hat{\mathbf{G}}_{e}^{H} \mathbf{G}\right]\left(\mathbf{u}(n)-\mathbf{u}_{\infty}\right)
$$

When the eigenvalues of $\hat{\mathbf{G}}_{e}^{H} \mathbf{G}$ are denoted by $\lambda_{N u}$, from the principal coordinates analysis presented by Elliott [2], the condition for the stability of the adaptive algorithm is given by 


$$
0<\alpha<\frac{2 R e\left(\lambda_{N u}\right)}{\left|\lambda_{N u}\right|^{2}} \text { for all } N u
$$

Therefore, a sufficient condition for the convergence is that the real parts of all the eigenvalues must be positive. In the case considered here, when the remote microphone method is combined with the filtered-reference LMS algorithm, and using the definition of the effective plant response, $\mathbf{G}$, above, the sufficient condition becomes [14]

$$
\operatorname{Re}\left(e i g\left[\hat{\mathbf{G}}_{\mathbf{e}}^{H} \hat{\mathbf{G}}_{\mathbf{e}}+\hat{\mathbf{G}}_{\mathbf{e}}^{H} \hat{\mathbf{O}}_{\mathrm{opt}}\left(\mathbf{G}_{\mathbf{m}}-\hat{\mathbf{G}}_{\mathbf{m}}\right)\right]\right)>0 .
$$

In Eq. (17), because the real parts of the eigenvalues of $\hat{\mathbf{G}}_{e}^{H} \hat{\mathbf{G}}_{e}$ are always positive, the stability is determined by the term, $\hat{\mathbf{G}}_{e}^{H} \hat{\mathbf{O}}_{\text {opt }}\left(\mathbf{G}_{m}-\hat{\mathbf{G}}_{m}\right)$. This would be zero if $\hat{\mathbf{G}}_{m}$ was exactly equal to $\mathbf{G}_{m}$, but in practice small differences between these matrices can be amplified by $\hat{\mathrm{O}}_{\text {opt }}$ if the elements of this observation filter are large.

When the sound at the ears of a listener is locally controlled, $\mathbf{G}_{m}, \mathbf{G}_{e}$ and $\mathbf{d}_{e}$ will all be modified by head movements. Therefore, if $\hat{\mathbf{G}}_{m}, \hat{\mathbf{G}}_{e}$ and $\hat{\mathbf{O}}_{\text {opt }}$ were pre-modelled and fixed at a nominal head position, the stability and performance of the active headrest system would be influenced by differences between the pre-modelled responses and the physical responses that will be introduced due to head movements. To overcome this problem, a head tracking device can be integrated with the active headrest system, which uses the remote microphone technique and adaptive control algorithm shown in Fig. 2. The integrated active headrest system is shown in Fig. 3. The head tracking device detects changes in the listener's head position and provides this information to the adaptive algorithm employing the remote microphone technique. The control system can then use this position information to update $\hat{\mathbf{G}}_{m}, \hat{\mathbf{G}}_{e}$ and $\hat{\mathbf{O}}_{\text {opt }}$ dynamically, as the head position is changed, by using a pre-calculated lookup table, for example.

\section{REAL-TIME IMPLEMENTATION AND EXPERIMENTAL INVESTIGA- TION}

To investigate the performance of the integrated active headrest system in a practical arrangement, several experiments have been conducted. In this section, the optimized observation filters have been calculated by off-line analysis of the measured acoustic transfer 


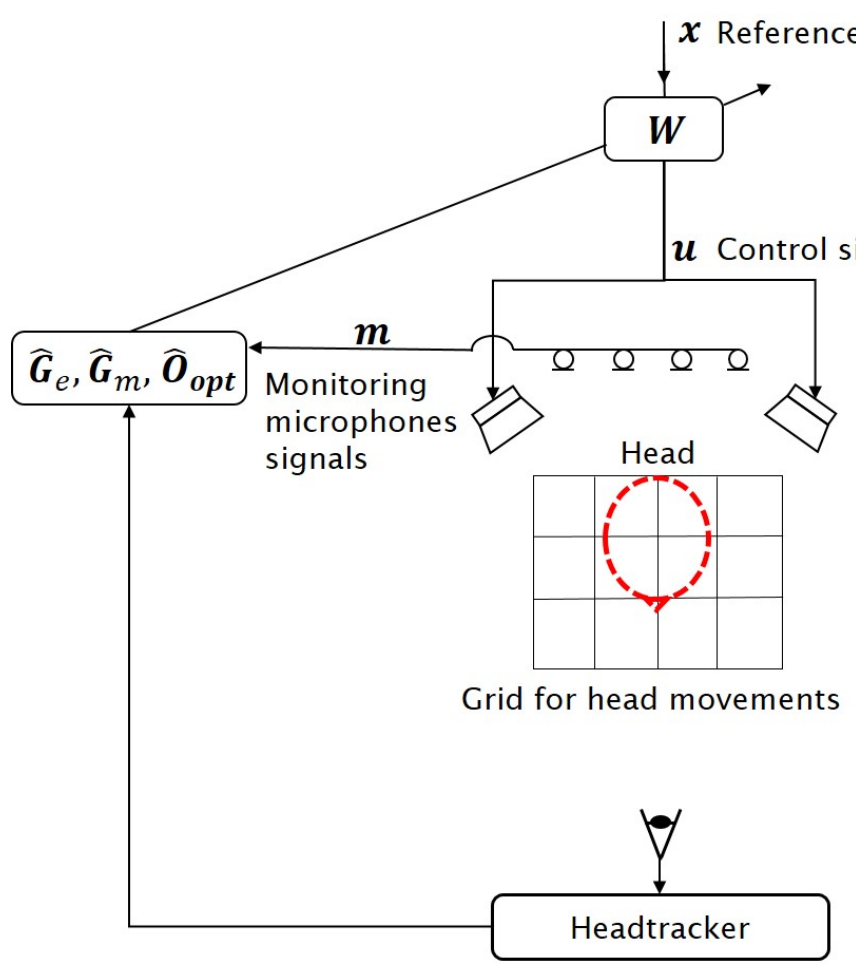

FIG. 3. The active headrest system integrated with the headtracker and monitoring microphones of the remote microphone technique.

responses, based on the theory developed in the previous section. Experiments have then been conducted to investigate the performance of the active headrest system using the remote microphone technique and head-tracking in real-time, when the primary sound field is produced by a single tonal source.

\section{A. Experiment arrangement}

Fig. 4 shows the experimental installation used for the real-time implementation and testing in an anechoic chamber. Four monitoring microphones were installed, with two on the back of the headrest and two on the supporting structure, as shown in Fig. 4. The positions of these monitoring microphones were selected through a series of preliminary experiments to obtain an accurate nearfield estimation of the disturbance signals at the virtual error sensors. To compare the estimated disturbance signals at the virtual error microphones with the actual disturbance signals, two microphones were also installed in the ears of a dummy head. The dummy head and physical error microphones can be removed 
after the preliminary measurements and the system can be used by a real listener. After calculating the observation filter, the active headrest system with the adaptive algorithm shown in Fig. 2 was applied using the measured plant responses, to reduce the disturbance signals in real-time. The full set of transfer responses between the primary and secondary sources and the monitoring and physical error microphones was measured when the dummy head was located at 20 different positions on a $5 \times 4$ grid of points spaced $5 \mathrm{~cm}$ apart. A single loudspeaker was installed behind the active headrest system to act as the primary source, driven by a single frequency signal. Finally, a commercial device, the Microsoft Kinect, was installed in front of the dummy head and used to track the head position. The information from the Kinect was decoded in real-time using plug-in software implemented in MaxMSP and this was passed to a dSPACE-based adaptive controller, which already had stored in it the various plant responses and pre-calculated observation filters for the 20 head positions.

\section{B. The nearfield estimation of tonal sounds in real-time}

As mentioned in Section II, the observation filter must be pre-calculated using Eq. (5) and the pre-measured acoustic responses. In addition, an appropriate regularization factor is necessary in order to achieve both accurate estimation and robustness to practical uncertainties. An estimate of the robustness of the system can be obtained from the condition number of the matrix being inverted in Eq. (5). Fig. 5 shows that for an excitation frequency of $600 \mathrm{~Hz}$, a regularization factor between $10^{-4}$ and $10^{-2}$ appears to give a reasonable tradeoff between the condition number and the estimation error if an attenuation of at least $10 \mathrm{~dB}$ is to be achieved. The estimation accuracy was calculated using the data measured in the installation shown in Fig. 4 with the disturbance signals at the error microphones estimated from the monitoring microphone array using the observation filters given by Eq. (5). The estimation error at each microphone, $E_{n}$, has been defined as

$$
E_{n}=10 \log _{10}\left|\frac{S_{\epsilon \epsilon, n}}{S_{d_{e} d_{e}, n}}\right|,
$$

where $n$ is the number of the error microphone, and $E_{1}$ and $E_{2}$ indicate respectively the estimation error at the right and left error microphones, and if $\epsilon$ is defined as $\mathbf{d}_{e}-\hat{\mathbf{d}}_{e}$, then 

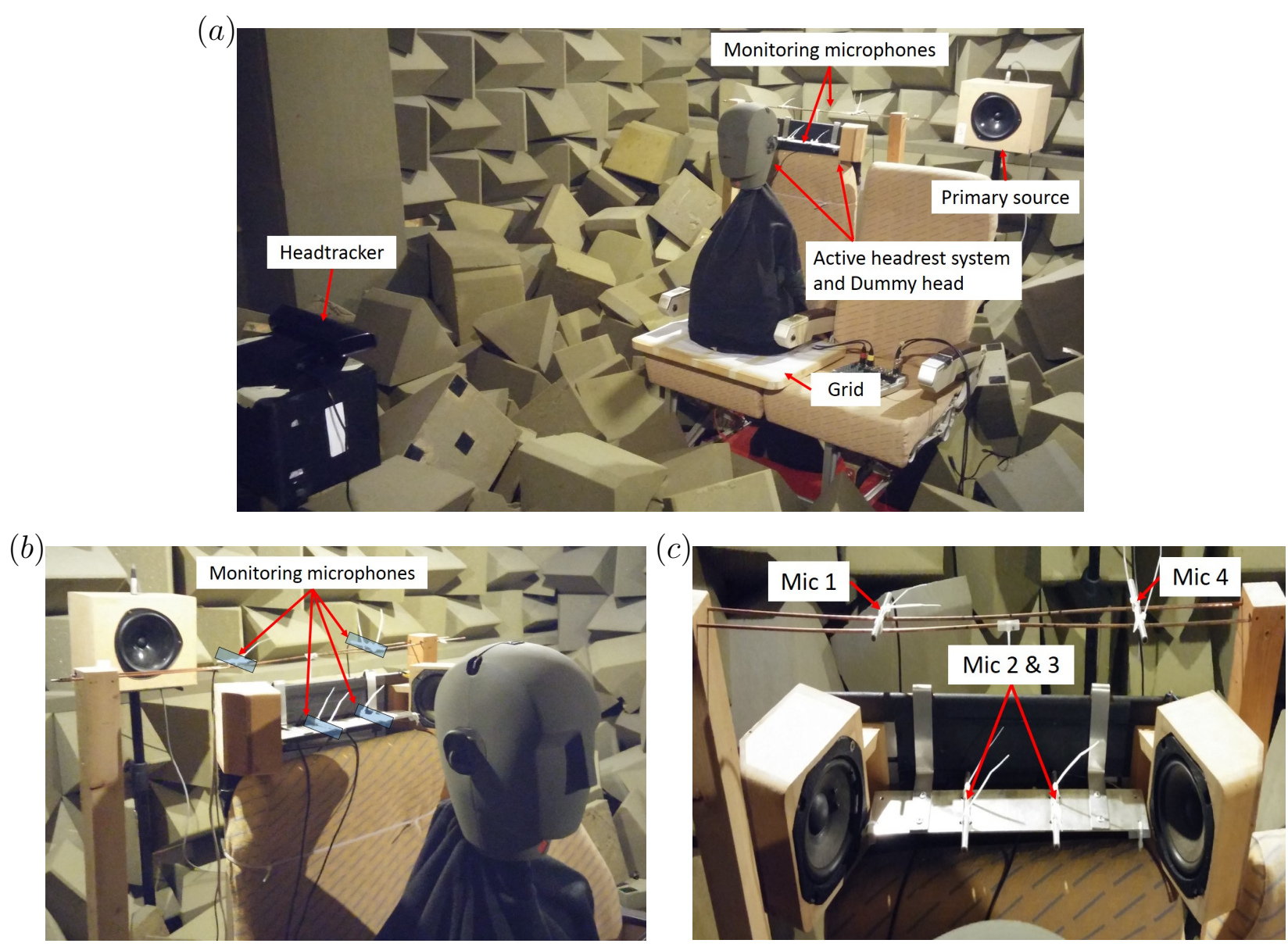

FIG. 4. (a) The overall installation for the real-time operation of both the nearfield estimation and the integrated active headrest system for controlling tonal disturbance signals, (b) the dummy head with 2 error microphones, one in each ear, and 4 monitoring microphones to the rear and (c) the monitoring microphones on both a mounting structure and the headrest.

$S_{d_{e} d_{e}, n}$ and $S_{\epsilon \epsilon, n}$ are the diagonal elements of the spectral density matrices, $\mathbf{S}_{d_{e} d_{e}}$ and $\mathbf{S}_{\epsilon \epsilon}$.

In order to determine an appropriate regularization factor for the real-time implementation, values in the range of $10^{-4}$ to $10^{-2}$ were investigated through off-line calculation of the estimation accuracy and the robustness to uncertainty. Following the related analysis presented by Elliott et al. [16], the acoustical uncertainty, $\Delta_{d_{m}}$ in the power spectral density matrix of $\mathbf{d}_{m}$ can be included in Eq. (5) as

$$
\mathbf{O}_{\mathrm{opt}}=\mathbf{S}_{d_{m} d_{e}}\left(\mathbf{S}_{d_{m} d_{m}}+\Delta_{d_{m}}+\beta \mathbf{I}\right)^{-1}
$$

where $\Delta_{d_{m}}$ is defined as 


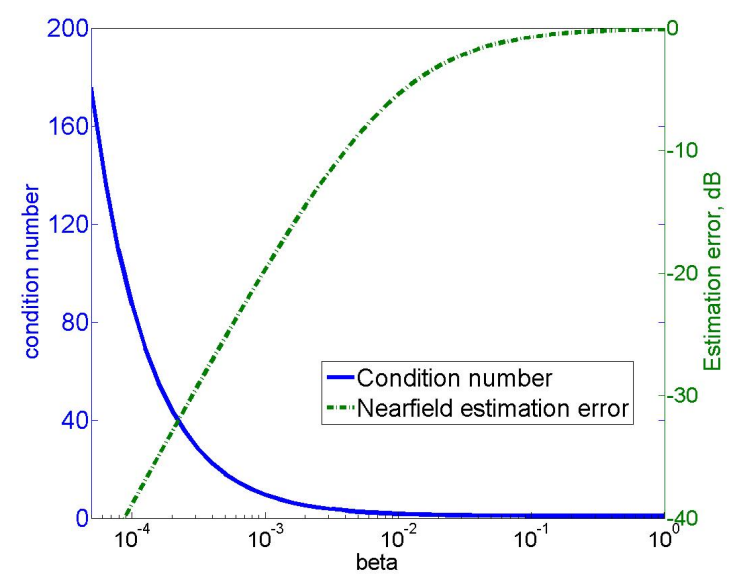

FIG. 5. Condition number of the inverse term in Eq. (5) (Solid line) and off-line nearfield estimation error (Dash-dot line) with different regularization factors to estimate the disturbance signals at the right error microphone at the nominal head position using the monitoring microphones at $600 \mathrm{~Hz}$.

$$
\Delta_{d_{m}}=e^{2} \frac{\left\|\mathbf{S}_{d_{m} d_{m}}\right\|_{F}}{M^{2}} \mathbf{I}
$$

where $e$ is the normalized rms error, \|\|$_{F}$ is the Frobenius matrix norm defined in [2] and $M$ is the number of monitoring microphones. The normalized rms error is set to either 0.05 , 0.1 or 0.2 , representing either $5 \%, 10 \%$, or $20 \%$ variation in the measured estimation of the pressure at the monitoring microphones. If different regularization factors are applied under these different levels of variation, changes in the nearfield estimation error indicate the robustness of the observation filter. Fig. 6 shows the nearfield estimation error for different levels of acoustical uncertainty for three different regularization factors. From the results in Fig. 6, it can be seen that when different levels of acoustical uncertainty exist, the nearfield estimation error with $\beta=10^{-4}$ is influenced by the acoustical uncertainty, although a reasonably accurate estimation is achievable even with $20 \%$ uncertainty. However, the influence of the acoustical uncertainty is decreased as the regularization factor is increased such that with $\beta=10^{-3}$ the variation in the estimation error for different levels of uncertainty is less than $1 \mathrm{~dB}$. From the results shown in Fig. 5 and Fig. 6 , a regularization factor, $\beta=10^{-3}$ was selected as appropriate for the real-time estimation. It can be seen from Fig. 6 that in the frequency range below $100 \mathrm{~Hz}$, the nearfield estimation error is significantly increased because the primary loudspeaker does not generate sound efficiently in this frequency range and so the measured pressures are dominated by background noise, giving poor coherence 
(a)

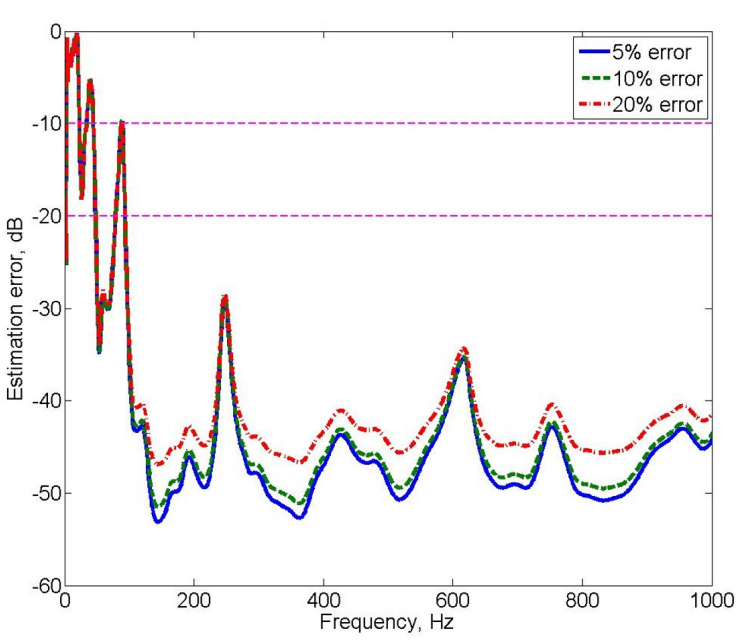

(b)

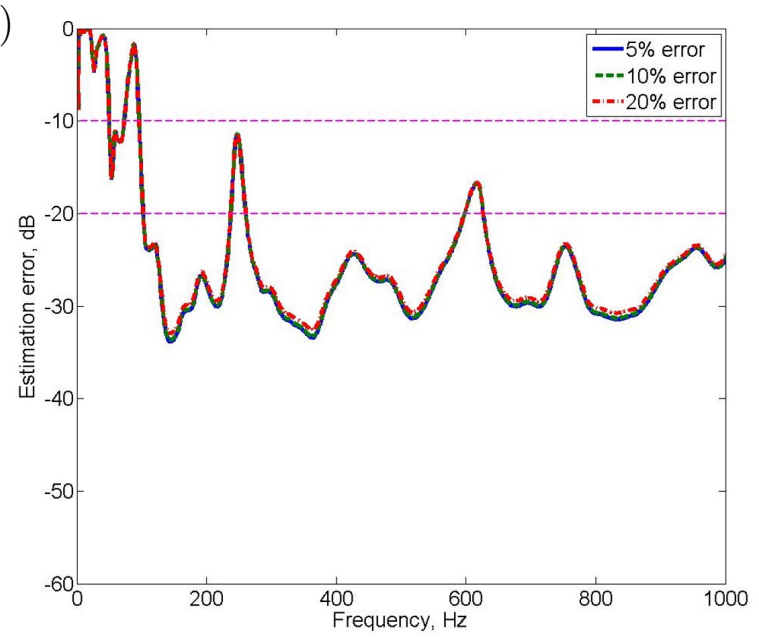

(c)

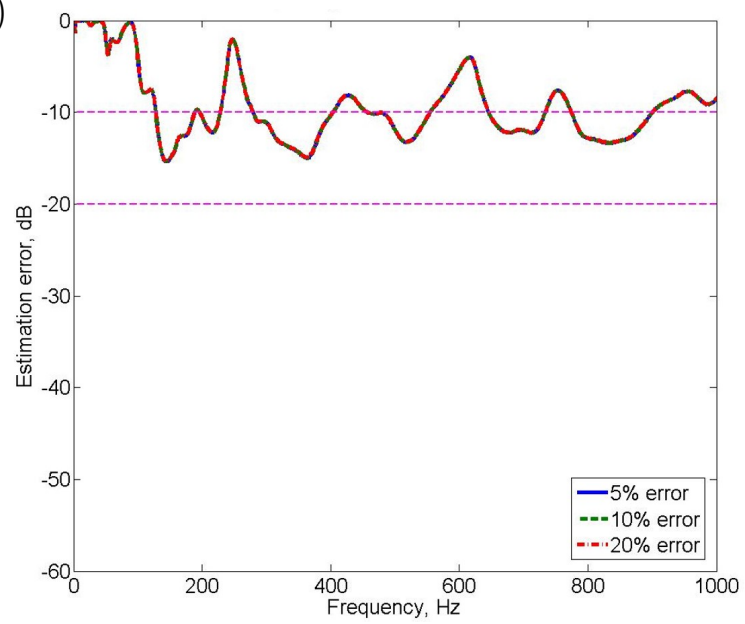

FIG. 6. Changes in the nearfield estimation error for different acoustic uncertainties in the analysis of the monitoring microphones when the observation filter is applied with different regularization factors as: (a) $\beta=10^{-4}$, (b) $\beta=10^{-3}$, (c) $\beta=10^{-2}$

between the loudspeaker input and microphone output.

When tonal disturbance signals at the dummy head error microphones at different frequencies are measured and compared with those estimated from the monitoring microphones in real-time, with the pre-calculated observation filter using a regularization of $\beta=10^{-3}$, results in Table I show that less than $-15 \mathrm{~dB}$ estimation error is achieved at all frequencies between 300 and $700 \mathrm{~Hz}$. Table I also shows that the off-line results, which are predicted from the measured acoustic responses, give a reasonable indication of the real-time performance. 


\section{Real-time adaptive control using the integrated active headrest system}

The real-time performance of the integrated active headrest system was then tested when a single primary source in the anechoic chamber was driven to produce a tonal disturbance. For the estimation of the virtual error signals, the observation filters described in the previous section, with a regularization factor of $\beta=10^{-3}$, were applied.

When the dummy head was located in the nominal position 'A' in Fig. 7, the attenuation performance was measured, as summarised in Table II. The attenuation of the estimated error signals inside the control system, i.e. $\hat{\mathbf{e}}(n)$ in Eq. (12), is termed the "predicted" attenuation in this table, and this is almost perfectly cancelled by the adaptive controller since this calculation takes no account of background noise on processing errors. The attenuation actually achieved at the microphones in the dummy head is degraded, because in the previous section, as the regularization factor was applied to improve the robustness of the observation filter, the nearfield estimation error between the actual and estimated error signals was increased to between -20 and $-30 \mathrm{~dB}$. If a smaller regularization factor for the observation filter is used, differences between the predicted and actual attenuation can be decreased. However, with $\beta=10^{-3}$, selected to giva a trade-off between the robustness and accuracy of the nearfield estimation, although the achieved performance is lower than the predicted performance, an actual attenuation of greater than $15 \mathrm{~dB}$ is still achieved at all frequencies between 300 and $700 \mathrm{~Hz}$. The real-time estimation error in Table I generally corresponds to the actual attenuation in Table II because when differences between $\mathbf{G}_{e}$ and $\hat{\mathbf{G}}_{e}$ and $\mathbf{G}_{m}$ and $\hat{\mathbf{G}}_{m}$ are negligibly small, the actual attenuation from Eq. (10) becomes similar to the nearfield estimation error in Eq. (18). Therefore, although the real-time adaptive control was implemented between 300 and $700 \mathrm{~Hz}$, a similar attenuation performance can be expected at higher frequencies based on the nearfield estimation error in Fig. 6.

The effect of the head-tracking device on the integrated active control system was then investigated by moving the dummy head from position 'A' to the different positions indicated in Fig. 7. Fig. 8 shows changes in the acoustic transfer responses, $\mathbf{G}_{e}, \mathbf{G}_{m}$ and $\mathbf{P}_{e}$ for different head positions. In Fig. 8, $\mathbf{G}_{e 11}$ and $\mathbf{G}_{e 12}$ indicate the acoustic transfer responses between the microphone in the right ear of the dummy head and the right and left secondary loudspeakers, respectively. In addition, $\mathbf{G}_{m 21}$ is the acoustic transfer response between the right secondary loudspeaker and monitoring microphone 2 and $\mathbf{P}_{e 11}$ is the acoustic transfer 


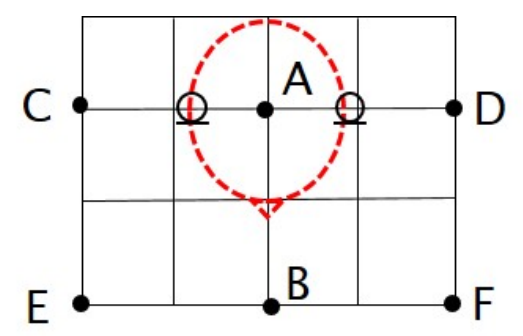

FIG. 7. Moved head positions on the grid.

response between the primary source and the right ear microphone. As the dummy head is moved, the acoustic responses, $\mathbf{G}_{e}$ and $\mathbf{P}_{e}$, which are directly related to the microphones in the dummy head, are significantly changed. It is also important to note, however, that the response from the secondary sources to the static monitoring microphones, $\mathbf{G}_{m}$ is also influenced by the head movement, due to the scattering effect of the dummy head. The use of the headtracker and the selection of accurate values for $\mathbf{G}_{e}, \mathbf{G}_{m}$ and $\mathbf{P}_{e}$ can thus significantly improve the attenuation performance and stability.

In Table III, attenuation results for the active headrest system with and without the headtracker in operation are compared for the 5 different positions, when the head-tracking device is used to update the acoustic transfer responses for the different positions, the attenuation performance is generally improved for all positions. In particular, when the dummy head is moved to either position ' $\mathrm{C}$ ', ' $\mathrm{D}$ ' or ' $\mathrm{E}$ ', noise enhancement, as indicated as negative attenuations, is generated without head-tracking at $700 \mathrm{~Hz}$, but the performance of the active headrest system with the headtracker is significantly improved. Since the error signals are influenced by the combined effects of a number of different terms, as seen in Eq. (10), it is difficult to pin down the source of the variability seen in Table III. Also, although in these experiments the control system remained stable as the dummy head was moved even without the head tracking, it was found in experiments at other frequencies that the control system became unstable without headtracking and that the control system could change from being stable to being unstable even for relatively small changes in the disturbance frequency.

Fig. 9 shows the time history of the signal measured by the microphone in the dummy head as it is moved from position 'A' to ' $\mathrm{D}$ ' for a disturbance frequency of either 600 $\mathrm{Hz}$ or $700 \mathrm{~Hz}$. The measured signals can be divided into three time intervals. The first 
(a)
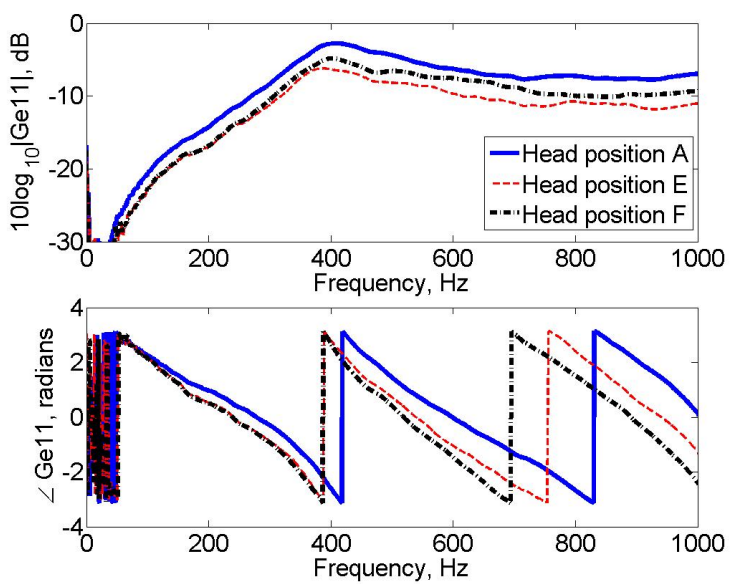

$(c)$
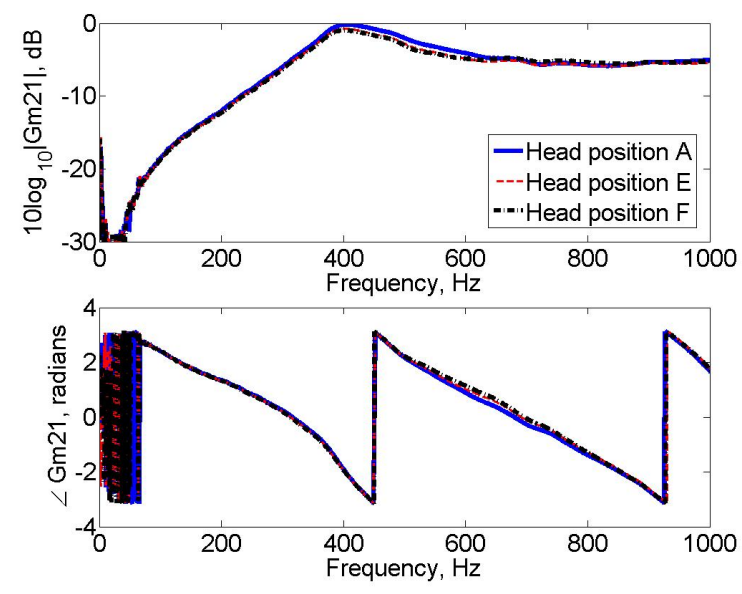

(b)
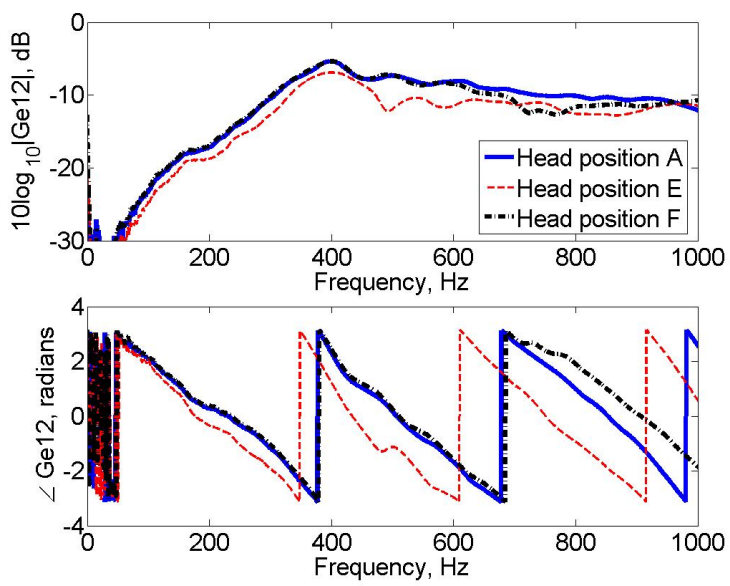

$(d)$
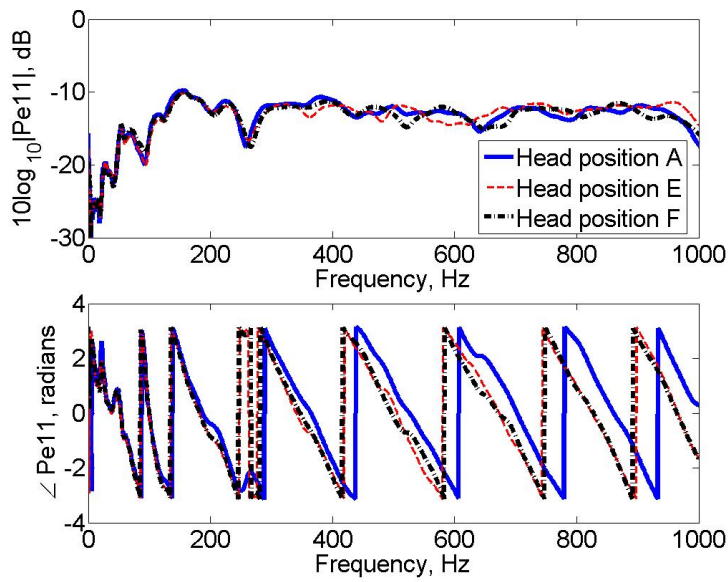

FIG. 8. Examples of changes in acoustic transfer responses, $\mathbf{G}_{e}, \mathbf{G}_{m}$ and $\mathbf{P}_{e}$ for different dummy head positions in Fig. 7: (a) $\mathbf{G}_{e 11}$ and (b) $\mathbf{G}_{e 12}$ between the right and left secondary loudspeakers and the right error microphone at dummy head, (c) $\mathbf{G}_{m 21}$ between the right secondary loudspeaker and the monitoring microphone 2 and (d) $\mathbf{P}_{e 11}$ between the primary source and the right ear microphone.

interval shows the disturbance signals without control when the dummy head is located at position 'D'. The second interval shows the error signals when the active headrest system is implemented without the head tracker. It can be seen that the disturbance signal at $600 \mathrm{~Hz}$ is slightly reduced but the disturbance signal at $700 \mathrm{~Hz}$ is enhanced due to the significant difference between the virtual and actual error signals. The head tracker is then in operation in the third time interval, and the attenuation performance is significantly improved at both frequencies.

The feasibility of this integrated active headrest system was also tested with a human 
(a)

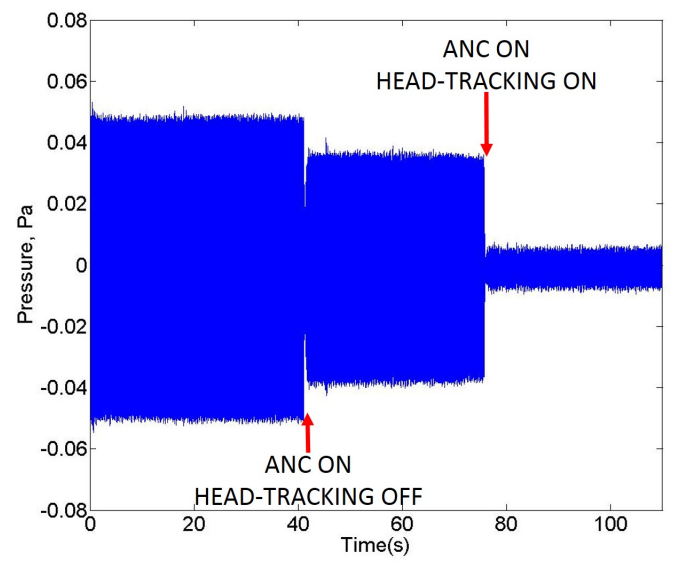

(b)

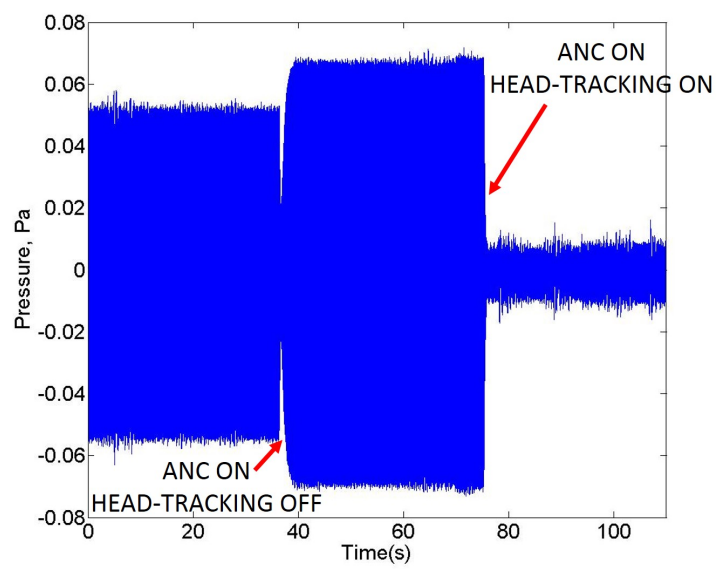

FIG. 9. Measured signals at the right error microphone of the dummy head at position ' $D$ ' when the integrated active headrest system with the remote microphone technique and the head tracker reduces the tonal disturbance signals from a single primary source in the anechoic chamber: (a) $600 \mathrm{~Hz}$, (b) $700 \mathrm{~Hz}$

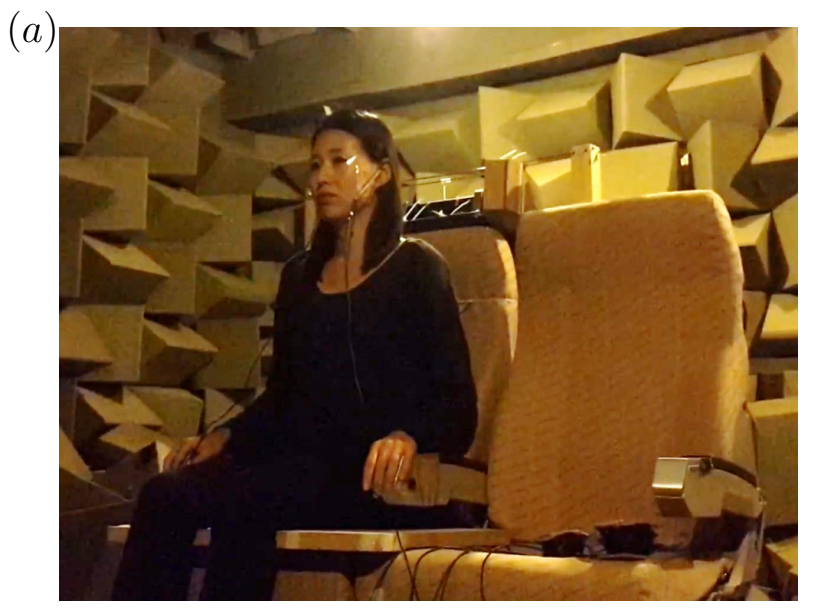

(b)

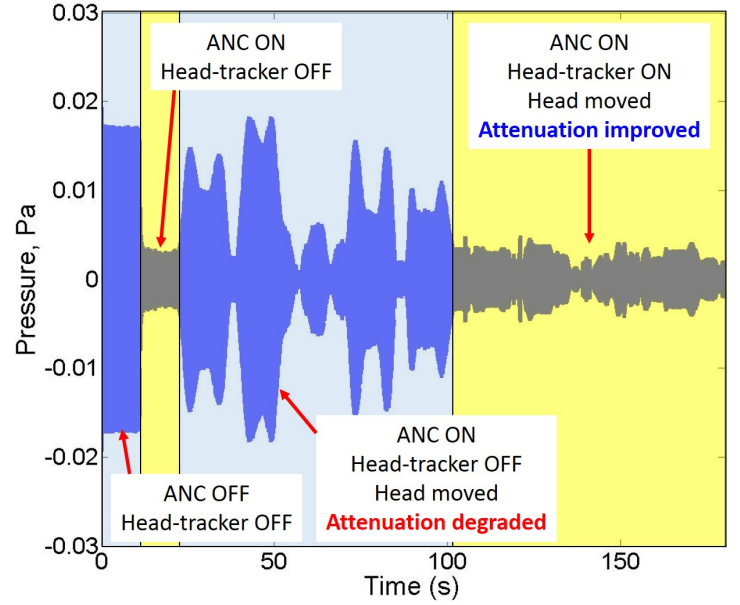

FIG. 10. Active control of the integrated active headrest system with a human listener for reducing $600 \mathrm{~Hz}$ tonal disturbance signals in real-time: (a) The test installation, in which the participant has two physical error microphones in their ears for evaluation purposes and (b) the measured signals at these error microphones during active control and head-tracking.

listener moving their head. The installation shown in Fig. 4 was again used, but two physical error microphones were installed, for evaluation purposes, at the two ears of the human participant instead of in the dummy head. The active control system was implemented to reduce a $600 \mathrm{~Hz}$ tonal disturbance signal and the participant moved to different positions similar to those shown in Fig. 7. The resulting signals measured at the physical error microphones are shown in Fig. 10. In the second time interval, from about 10 to 20 seconds, 
the active headrest system is switched on and effectively attenuates the disturbance signals at the nominal position. However, it can be seen from the third time interval, from about 20 to 100 seconds, that as the listener then moves their head to different positions without the headtracker, the performance of the control system is significantly degraded. In the final step, from about 100 to 180 seconds, the headtracker is switched on and the control system achieves an improved performance in spite of head movements. In a practical system the accuracy and speed of the response of the headtracker may be important, depending on the application, but the current system demonstrates the feasibility with a commercially available device, the Kinect, that was not specifically designed for this purpose.

\section{CONCLUSIONS}

The integration of both the remote microphone technique and head-tracking into an active headrest system has been investigated. The remote microphone technique has been formulated in the frequency domain and equations for the observation filter have been discussed. When the remote microphone technique is combined with an adaptive feedforward active control system, equations for the optimal attenuation performance and for the stability have also been derived. In this combined system, the use of a head-tracking device is investigated to improve the performance and stability when the head of a listener is moving.

The nearfield estimation achieved using the observation filter and the integration of the remote microphone technique and head-tracking have been verified through real-time experiments, when a single primary source produces tonal noise in an anechoic chamber. To find an appropriate regularization factor for the observation filter calculation, changes in the accuracy and robustness of the nearfield estimation with different regularization factors have been calculated. With an observation filter having the selected regularization factor, the signals at the ears of a dummy head can be estimated in real-time from remotely installed monitoring microphones with less than $-15 \mathrm{~dB}$ error. The adaptive active control system combined with the remote microphone technique also achieved more than $15 \mathrm{~dB}$ attenuation of the signals measured at the ears of the dummy head, which is located at a fixed nominal head position, for frequencies up to $700 \mathrm{~Hz}$. When the dummy head was moved to different positions, the attenuation was degraded unless a headtracking device was used to update the acoustic responses using a look-up table containing pre-modelled responses. 


\section{ACKNOWLEDGMENTS}

This research is jointly funded by an EPSRC industrial CASE studentship (Award no. 14220108) with Jaguar Land Rover (JLR). The authors are especially thankful to Dr Delphine Nourzad of JLR for her support as an industrial supervisor.

[1] P. A. Nelson and S. J. Elliott, Active Control of Sound, (Academic Press, London, 1992), Chap. 9\&10, pp. 310-378.

[2] S. J. Elliott, Signal Processing for Active Control, (Academic Press, London, 2000), Chap.1, pp. 33-48.

[3] C. H. Hansen, S. D. Snyder, X. Qiu, L. Brooks and D. Moreau, Active Control of Noise and Vibration Volume II, (CRC Press, USA, 2012), Chap. 9, pp. 983-1092.

[4] B. Rafaely, S. J. Elliott and J. Garcia-Bonito, "Broadband performance of an active headrest", J. Acoust. Soc. Am. 106(2), 787-793 (1999).

[5] M. Pawelczyk, "Adaptive noise control algorithms for active headrest system", Control Eng. Pract. 12(9), 1101-1112 (2004).

[6] D. P. Das, D. J. Moreau and B. Cazzolato, "Performance evaluation of an active headrest using the remote microphone technique", in Proc. Acoustics 2011, Gold Coast, 69-76 (2011).

[7] A. Siswanto, C. Y. Chang and S. M. Kuo, "Active noise control for headrests", in Proc. APSIPA Annual Summit and Conference 2015, Hong Kong, 688-692 (2015).

[8] S. J. Elliott, P. Joseph, A. J. Bullmore and P. A. Nelson, "Active cancellation at a point in a pure tone diffuse sound field", J. Sound Vib. 120(1), 183-189 (1988).

[9] A. Roure and A. Albarrazin, "The remote microphone technique for active noise control", in Proc. INTER-NOISE and NOISE-CON Congress and Conference, 5, 1233-1244 (1999).

[10] D. Moreau, B. Cazzolato, A. Zander and C. Petersen, "A review of virtual sensing algorithms for active noise control", Algorithms 1(2), 69-99 (2008).

[11] S. J. Elliott and J. Cheer, "Modelling local active sound control with remote sensors in spatially 
random pressure fields", J. Acoust. Soc. Am. 137(4), 1936-1946 (2015).

[12] D. J. Moreau, B. S. Cazzolato and A. C. Zander, "Active noise control at a moving virtual microphone using the SOTDF moving virtual sensing method", in Proc. Acoustics 2009, Adelaide, (2009).

[13] S. K. Behera, D. P. Das and B. Subudhi, "Active headrest with moving error microphone for real-time adaptive noise control", in Control Conference (ICC), 2017, Guwahati, 356-363 (2017).

[14] S. J. Elliott, M. Simon, J. Cheer and W. Jung, "Head tracking for local active noise control", in Proc. WESPAC, 2015, Singapore, 385-389 (2015).

[15] W. Jung, S. J. Elliott and J. Cheer, "The effect of remote microphone technique and headtracking on local active sound control", in Proc. ICSV23, Athens, (2016).

[16] S. J. Elliott, J. Cheer, J. W. Choi and Y. Kim "Robustness and regularization of personal audio systems", IEEE Trans. Audio, Speech, Language Process. 20(7). 2123-2133 (2012). 
Fig. 1. Block diagram of the remote microphone technique.

Fig. 2. Block diagram of the filtered-reference LMS algorithm for adaptive feedforward control, combined with the remote microphone technique.

Fig. 3. The active headrest system integrated with the headtracker and monitoring microphones of the remote microphone technique.

Fig. 4. The overall installation for the real-time operation of both the nearfield estimation and the integrated active headrest system for controlling tonal disturbance signals, (b) the dummy head with 2 error microphones, one in each ear, and 4 monitoring microphones to the rear and (c) the monitoring microphones on both a mounting structure and the headrest.

Fig. 5. Condition number of the inverse term in Eq. (5) (Solid line) and off-line nearfield estimation error (Dash-dot line) with different regularization factors to estimate the disturbance signals at the right error microphone at the nominal head position using the monitoring microphones at $600 \mathrm{~Hz}$.

Fig. 6. Changes in the nearfield estimation error for different acoustic uncertainties in the analysis of the monitoring microphones when the observation filter is applied with different regularization factors as: (a) $\beta=10^{-4}$, (b) $\beta=10^{-3}$, (c) $\beta=10^{-2}$

Fig. 7. Moved head positions on the grid.

Fig. 8. Examples of changes in acoustic transfer responses, $\mathbf{G}_{e}, \mathbf{G}_{m}$ and $\mathbf{P}_{e}$ for different dummy head positions in Fig. 7: (a) $\mathbf{G}_{e 11}$ and (b) $\mathbf{G}_{e 12}$ between the right and left secondary loudspeakers and the right error microphone at dummy head, (c) $\mathbf{G}_{m 21}$ between the right secondary loudspeaker and the monitoring microphone 2 and (d) $\mathbf{P}_{e 11}$ between the primary source and the right ear microphone.

Fig. 9. Measured signals at the right error microphone of the dummy head at position ' $\mathrm{D}$ ' when the integrated active headrest system with the remote microphone technique and the head tracker reduces the tonal disturbance signals from a single primary source in the anechoic chamber: (a) $600 \mathrm{~Hz}$, (b) $700 \mathrm{~Hz}$ 
Fig. 10. Active control of the integrated active headrest system with a human listener for reducing $600 \mathrm{~Hz}$ tonal disturbance signals in real-time: (a) The test installation, in which the participant has two physical error microphones in their ears for evaluation purposes and (b) the measured signals at these error microphones during active control and head-tracking. 
TABLE I. Comparisons of the nearfield estimation error between the off-line and real-time test when tonal disturbance signals at the right error microphone of the dummy head are estimated by the 4 monitoring microphones around the headrest with the pre-calculated observation filter in the anechoic chamber.

\begin{tabular}{|c|c|c|}
\hline Frequencies & $\begin{array}{c}\text { Off-line } \\
\text { estimation error }(\mathrm{dB})\end{array}$ & $\begin{array}{c}\text { Real-time estimation error } \\
\text { Magnitude }(\mathrm{dB}) \text {, Phase (radians) }\end{array}$ \\
\hline $300 \mathrm{~Hz}$ & -28.5 & $-29.4,-0.01 \pi$ \\
\hline $400 \mathrm{~Hz}$ & -28.9 & $-32.1,-0.01 \pi$ \\
\hline $500 \mathrm{~Hz}$ & -27.7 & $-19.3,-0.03 \pi$ \\
\hline $600 \mathrm{~Hz}$ & -22.9 & $-21.1,-0.02 \pi$ \\
\hline $700 \mathrm{~Hz}$ & -27.8 & $-17.0,-0.01 \pi$ \\
\hline
\end{tabular}

TABLE II. Predicted and achieved attenuation performance of the integrated active headrest system for tonal disturbances at different frequencies in the anechoic chamber when the dummy head is located in position ' $\mathrm{A}$ '.

\begin{tabular}{|c|c|c|}
\hline Frequencies & $\begin{array}{c}\text { Predicted attenuation } \\
\text { right / left ear, }(\mathrm{dB})\end{array}$ & $\begin{array}{c}\text { Achieved attenuation } \\
\text { right / left ear, }(\mathrm{dB})\end{array}$ \\
\hline $300 \mathrm{~Hz}$ & $75.9 / 78.0$ & $22.4 / 26.1$ \\
\hline $400 \mathrm{~Hz}$ & $94.2 / 94.8$ & $23.3 / 30.3$ \\
\hline $500 \mathrm{~Hz}$ & $99.1 / 98.3$ & $23.9 / 20.4$ \\
\hline $600 \mathrm{~Hz}$ & $82.0 / 80.7$ & $15.9 / 28.6$ \\
\hline $700 \mathrm{~Hz}$ & $87.1 / 87.9$ & $15.0 / 26.0$ \\
\hline
\end{tabular}


TABLE III. Comparison between the actual attenuation performance of the integrated active headrest system with and without the head-tracking system when the dummy head is moved to different positions and a single loudspeaker produces a tonal disturbance at different frequencies in the anechoic chamber.

\begin{tabular}{|c|c|c|c|c|}
\hline \multirow{2}{*}{ Frequencies } & $\begin{array}{c}\text { Position 'B', Actual attenuation, } \\
\text { right/left ear }(\mathrm{dB})\end{array}$ & $\begin{array}{r}\text { Position 'C', Actual attenuation, } \\
\text { right/left ear }(\mathrm{dB})\end{array}$ \\
\cline { 2 - 5 } & $\begin{array}{c}\text { without } \\
\text { head-tracking }\end{array}$ & $\begin{array}{c}\text { with } \\
\text { head-tracking }\end{array}$ & $\begin{array}{c}\text { without } \\
\text { head-tracking }\end{array}$ & $\begin{array}{c}\text { with } \\
\text { head-tracking }\end{array}$ \\
\hline $300 \mathrm{~Hz}$ & $10.8 / 17.8$ & $21.1 / 23.1$ & $12.7 / 22.3$ & $27.0 / 22.0$ \\
\hline $400 \mathrm{~Hz}$ & $9.7 / 12.8$ & $18.5 / 16.6$ & $9.8 / 16.1$ & $16.8 / 15.6$ \\
\hline $500 \mathrm{~Hz}$ & $8.0 / 1.0$ & $30.2 / 4.5$ & $7.2 / 3.6$ & $14.0 / 11.0$ \\
\hline $600 \mathrm{~Hz}$ & $24.5 / 6.9$ & $13.7 / 13.0$ & $5.0 / 8.8$ & $17.6 / 11.6$ \\
\hline $700 \mathrm{~Hz}$ & $9.7 / 8.6$ & $21.0 / 11.4$ & $2.1 /-1.6$ & $17.3 / 9.3$ \\
\hline
\end{tabular}

\begin{tabular}{|c|c|c|c|c|}
\hline \multirow{2}{*}{ Frequencies } & $\begin{array}{r}\text { Position 'D', Actual attenuation, } \\
\text { right/left ear }(\mathrm{dB})\end{array}$ & $\begin{array}{r}\text { Position 'E', Actual attenuation, } \\
\text { right/left ear }(\mathrm{dB})\end{array}$ \\
\cline { 2 - 5 } & $\begin{array}{c}\text { without } \\
\text { head-tracking }\end{array}$ & $\begin{array}{c}\text { with } \\
\text { head-tracking }\end{array}$ & $\begin{array}{c}\text { without } \\
\text { head-tracking }\end{array}$ & $\begin{array}{c}\text { with } \\
\text { head-tracking }\end{array}$ \\
\hline $300 \mathrm{~Hz}$ & $19.4 / 11.8$ & $26.7 / 24.8$ & $7.9 / 20.6$ & $27.6 / 22.9$ \\
\hline $400 \mathrm{~Hz}$ & $14.1 / 5.7$ & $21.4 / 20.7$ & $5.0 / 14.2$ & $14.1 / 15.6$ \\
\hline $500 \mathrm{~Hz}$ & $0.9 / 6.4$ & $16.1 / 21.5$ & $5.1 / 3.2$ & $14.8 / 14.2$ \\
\hline $600 \mathrm{~Hz}$ & $2.5 / 4.0$ & $20.4 / 23.7$ & $5.3 / 19.5$ & $12.2 / 13.6$ \\
\hline $700 \mathrm{~Hz}$ & $-2.5 / 4.6$ & $16.9 / 23.2$ & $4.0 /-0.6$ & $22.1 / 16.0$ \\
\hline
\end{tabular}

\begin{tabular}{|c|c|c|}
\hline \multirow{2}{*}{ Frequencies } & $\begin{array}{c}\text { Position ' } \mathrm{r} \text { ', Actual attenuation, } \\
\text { right/left ear }(\mathrm{dB})\end{array}$ \\
\cline { 2 - 3 } & $\begin{array}{c}\text { without } \\
\text { head-tracking }\end{array}$ & $\begin{array}{c}\text { with } \\
\text { head-tracking }\end{array}$ \\
\hline $300 \mathrm{~Hz}$ & $21.5 / 12.3$ & $28.8 / 25.2$ \\
\hline $400 \mathrm{~Hz}$ & $15.0 / 6.1$ & $16.1 / 14.1$ \\
\hline $500 \mathrm{~Hz}$ & $4.3 / 14.3$ & $22.8 / 9.5$ \\
\hline $600 \mathrm{~Hz}$ & $13.2 / 1.1$ & $11.0 / 19.1$ \\
\hline $700 \mathrm{~Hz}$ & $0.8 / 3.4$ & $39.7 / 18.9$ \\
\hline
\end{tabular}

